\title{
VizCraft: A Multidimensional Visualization Tool for Aircraft Configuration Design
}

\author{
A. Goel ${ }^{*}$, C. Baker ${ }^{\dagger}$, C.A. Shaffer*, B. Grossman ${ }^{\dagger}$, R.T. Haftka ${ }^{\S}$, W.H. Mason ${ }^{\dagger}$, L.T. Watson* ${ }^{*}$ \\ ${ }^{*}$ Department of Computer Science, \\ ${ }^{\dagger}$ Multidisciplinary Analysis and Design Center for Advanced Vehicles, \\ Virginia Tech, Blacksburg VA 24061 \\ ${ }^{\S}$ Department of Aerospace Engineering, \\ Mechanics and Engineering Science, \\ University of Florida, Gainesville FL 32611
}

\begin{abstract}
We describe a visualization tool to aid aircraft designers during the conceptual design stage. The conceptual design for an aircraft is defined by a vector of $10-30$ parameters. The goal is to find a vector that minimizes an objective function while meeting a series of constraints. VizCraft integrates the simulation code that evaluates the design with visualizations for analyzing the design individually or in contrast to other designs. VizCraft allows the designer to easily switch between the view of a design in the form of a parameter set, and a visualization of the corresponding aircraft. The user can easily see which, if any, constraints are violated. VizCraft also allows the user to view a database of designs using parallel coordinates.
\end{abstract}

KEYWORDS: Scientific data visualization, aircraft design, multidisciplinary design optimization, multidimensional visualization.

\section{INTRODUCTION}

We describe a visualization tool to aid aircraft designers during the conceptual design stage. Typically, the aircraft design process is comprised of three distinct phases: conceptual, preliminary, and detailed design. In the conceptual design stage, major design parameters for the final configuration are defined and set. The conceptual design phase models an aircraft with a set of values for significant parameters, relating to the aircraft geometry, internal structure, systems, and mission. Examples of such parameters include the wing span, sweep, and thickness; the fuel and wing weights; the engine thrust; and the cruise altitude and climb rate.

Individual designs can be (and are) viewed as points in a multidimensional design space. The High-Speed Civil Transport (HSCT) [2] uses a design space with as many as 29 param-

0-7803-5897-X/99/\$10.00 Copyright 1999 IEEE eters. Two important features must be determined for any proposed design point: (1) it is feasible if it satisfies a series of constraints; and (2) it has a figure of merit determined by an objective function. The goal is then to find the feasible point with the smallest objective function value. In the HSCT design, take-off gross weight (TOGW) is chosen as the objective function because it is a rough indicator of the cost of the aircraft. Constraints are organized into two groups: geometric constraints versus aerodynamic/performance constraints. Examples of geometric constraints include fuel volume limits and prevention of tip strike at landing with $5^{\circ}$ roll. Examples of aerodynamic constraints include range requirements, landing angle of attack limits, and criteria to prevent wing and tail runway scrape.

In some respects, this is a classic optimization problem. The goal is to find that point which minimizes an objective function while meeting a series of constraints. However, this particular problem is difficult to solve for several reasons. First, evaluating an individual point to determine its value under the objective function and check if it satisfies the constraints is computationally expensive. A single aerodynamic analysis using a CFD code can take from $1 / 2$ hour to several hours, depending on the grid used and flight condition considered. Second, the high dimensionality of the problem makes it impractical for many approaches that are often applied to difficult optimization problems. The high dimensionality makes visualization of the design space difficult, since most standard visualization techniques do not apply. See $[6,5]$ for a more detailed description of the design problem and our initial efforts to gain some insight into the design space through visualization.

We next describe VizCraft, a pair of tools for visualizing HSCT designs. The first tool permits the user to quickly evaluate the quality of a given design with respect to its objective function, constraint violations, and graphical view. The second tool is an implementation of the parallel coordinates visualization [3]. Its goal is to allow the user to effectively investigate a database of designs. 


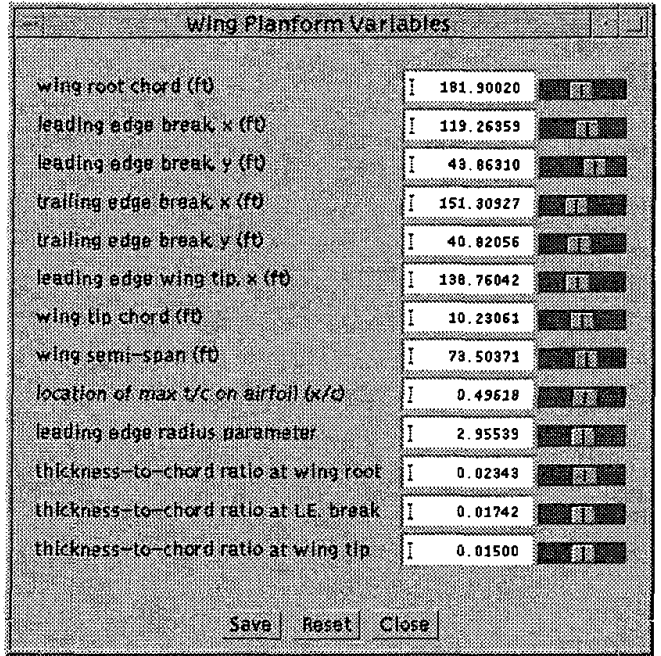

Figure 1. Wing planform variables.

\section{DESIGN POINT VISUALIZATION}

VizCraft provides a menu-driven graphical user interface to the HSCT design code, which is a collection of $\mathrm{C}$ and Fortran routines that calculate the aircraft geometry in 3-D, the design constraint values, and the TOGW value, among other things. Figure $\mathrm{C} 1$ shows VizCraft's main window with a display of the HSCT planform (a top view) for a sample design. Below the planform are displayed cross-sections of the airfoil at the root, leading edge break, and tip of the wing, in that order. We also added a VRML model of the HSCT planform, accessible from the menu bar.

Clicking on the "Wing Planform" button in the main window brings up the window shown in Figure 1. This window displays the wing parameters and the values currently assigned to them. The sliders on the right can be used to modify the values of the corresponding design variables. Each time the value of a design variable is modified, the HSCT planform is immediately updated to reflect the new geometry, and so is the value of TOGW on the vertical panel. Constraints for the current design point are not automatically evaluated after each change to an input parameter, however. Since constraint evaluation is time-consuming even for the low fidelity model we are using (taking approximately 10 seconds on a dual-processor DEC Alpha 4100 5/400 under typical loads), VizCraft evaluates constraints only when the user explicitly requests it by clicking on the "Evaluate" button shown in Figure $\mathrm{C} 1$.

Once constraints are evaluated, the user is given feedback in various ways. The color boxes shown in Figure $\mathrm{C} 1$ represent information about the number of constraints violated, nearly violated, and satisfied but inactive, in each category of constraints. The red boxes indicate the number of constraints of that category that are violated, the yellow boxes indicate the number of constraints that are "active," (i.e., close to a con-

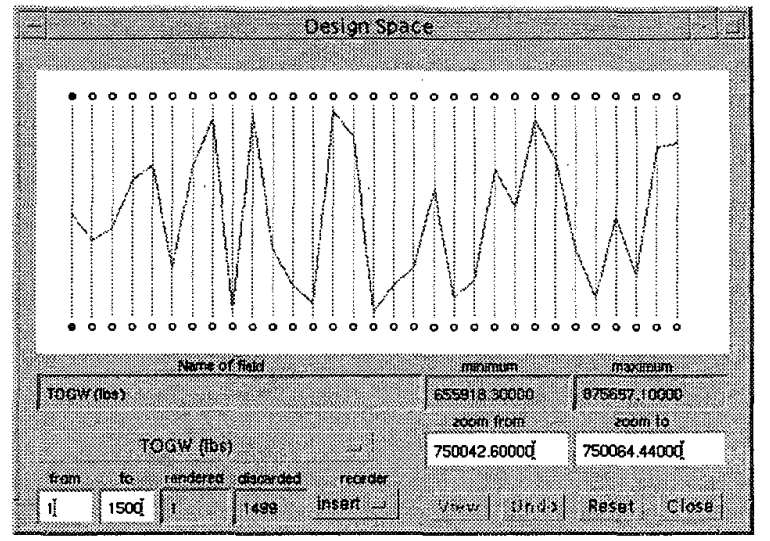

Figure 2. Parallel coordinates representation of one design point.

straint boundary), and the green boxes indicate the number of constraints that are inactive. Clicking on the "Geometric" Constraints button brings up the window shown in Figure C2. This window lists the geometric constraints for the current design point, and a color box next to each one indicates if it is violated, active, or inactive.

\section{PARALLEL COORDINATES}

The tool described in the previous section provides a visualization of the aircraft that would be derived from a given design vector, and also provides a convenient view of constraint violations. However, it does not help designers with the more difficult task of understanding how a proposed design compares with other designs. This task is complicated by the high dimensionality of the design problem, and the resulting difficulty in visualizing or comprehending the multidimensional design space. Few visualization techniques provide an adequate visualization of high-dimensional spaces.

One method of visualizing multiple dimensions is based on the concept of parallel coordinates [3]. A parallel coordinates visualization assigns one vertical axis to each visualization variable, and evenly spaces these axes horizontally. In our application, potential visualization variables include the design variables, the objective function value (TOGW) and other derived values such as range, and the constraint values. Each visualization variable is plotted on its own axis, and the values of the variables on adjacent axes are connected by straight lines, as shown in Figure 2 . Thus, a point in an $n$ dimensional space becomes a polygonal line laid out across the $n$ parallel axes with $n-1$ line segments connecting the $n$ data values. Many such data points (in Euclidean space) will map to many of these polygonal lines in a parallel coordinate representation. Viewed as a whole, these many lines hopefully will exhibit coherent patterns which could be associated with inherent correlation of the data points involved. In this way, the search for relations among the design variables is transformed into a 2-D pattern recognition problem, 
and the design points become amenable to visualization.

One important aspect of this visualization scheme is that it provides opportunities for human pattern recognition: By using color to distinguish lines, and by supporting various forms of interaction with the parallel coordinates system, patterns can be picked up from the database of design points. Given the upper and lower limits on each variable, the location of a polygonal line laid out across the $n$ vertical axes gives some idea as to where that design point lies in the design space. The number of dimensions that can be visualized using this scheme is fairly large, limited only by the horizontal resolution of the screen.

In Figure 2, 31 values are shown mapped onto 31 vertical axes. The first axis represents the TOGW, the second represents the HSCT range, and the remaining 29 axes represent the 29 design variables. Placing the mouse cursor on one of the circles below the vertical lines will cause the "Name of field" text field to display a description of the corresponding visualization variable. Figure C3 shows the parallel coordinates system for 68 constraints corresponding to the design point shown in Figure 2. All values above the yellow horizontal line indicate inactive constraints, all values between the yellow and red lines indicate active constraints, and all values below the red horizontal line indicate violated constraints. By breaking up the range of constraint values into three regions, it becomes easy to identify graphically the inactive and violated constraints, and to what degree each constraint has been violated.

Representing just one design point in the parallel coordinates system may help the designer quickly view the level of constraint violations, but this is little better than the view provided by the single-point VizCraft tool. The real purpose of parallel coordinates in VizCraft is to allow the designer to browse a database of design points. We illustrate this process with a database of 1500 design points selected uniformly from the entire design space. When this database is rendered using parallel coordinates, it appears as shown in Figure C4. From this mass of data, one can use VizCraft's visualization controls to extract patterns.

Each polygonal line (representing one design point) is assigned a color based on the value of a particular visualization parameter. In Figure $\mathrm{C} 4$, the value used to determine the color is TOGW. Thus, as lines span across the vertical axes, one can identify those design points for which the TOGW is high or low. The design point with lowest value of TOGW is assigned a yellow color, the one with the highest value is assigned a black color, while the color for all the other design points is a linear interpolation between yellow and black. Since the design objective is to minimize the TOGW, the designer might initially be interested in lines rendered in yellow. However, it may prove equally useful to the designer to discover that certain design variable ranges are associated with bad designs as it is to discover that other ranges are as- sociated with good designs.

Looking at Figure C4, one can already see from the color gradation that the sixth axis from the right is directly related to the first axis. It so happens that the sixth axis from the right represents the weight of the flight fuel in lbs, which affects the TOGW directly. One can also observe that the second axis from the left is also mildly correlated to the TOGW and flight fuel. This axis represents the range of the aircraft in nautical miles, which must be directly proportional to the amount of fuel added. Even though these particular relationships are obvious (once the viewer has an understanding of the parameters involved), they give us a good start into understanding how to extract patterns from the data.

\section{VISUAL DATA MINING}

A display of the full database such as shown in Figure C4 is typically too overwhelming to gain any real understanding of the data. The real strength of the parallel coordinates tool in VizCraft is the capability it provides for exploring the database. In this section we explain how the user can interact with the system "visual cues" [4] that will help in visualizing the data set in $n$-dimensional space.

Looking at Figure C4, notice that there is a circle above each vertical axis, and that only the first one on the left is shaded. The shaded circle indicates the visualization variable that is currently "driving" the gradation of color across the parallel coordinates. In Figure C4, TOGW is driving the color gradation. The user can select any visualization variable to drive the coloring by clicking inside the circle over the corresponding variable's axis. Clicking on the fifth circle we see that that variable happens to share a direct relationship with the seventh visualization variable (Figure C5). This shows that a clever selection of color drivers can help us extract patterns from the data set - patterns which are otherwise hidden underneath the volume of data.

The user's ability to recognize patterns in the parallel coordinates representation can be greatly affected by the sequence in which the axes are placed. For example, it is easier to perceive relationships between two adjacent axes than if the two axes are placed far apart. VizCraft allows the user to rearrange the axes. The user simply clicks on the circle above the axis to be moved and drags it to the new position. This rearrangement must be done with the re-order option set to "insert". If the re-order option is set to "swap", then one axis can be swapped with another by clicking and dragging one circle onto another. See [1] for a discussion on automating the process of initially arranging the axes to maximize similarities of adjacent axes.

While showing a large number of design points can be helpful in generating patterns that may be of interest to the researcher at a holistic level, individual design points cannot be distinguished when too many are displayed at once. To allow clear views of individual design points, the user may 
wish to select from this design space a sub-region of interest, or a sub-region that meets certain criteria. For example, the user may wish to eliminate all design points for which TOGW is greater than $700,000 \mathrm{lbs}$, or eliminate those points for which the range of the aircraft is less than 4,000 miles. The goal is to allow the user to gain some understanding of spatial relationships in $n$-space by selecting all data points that fall within a user-defined set. This technique of graphically selecting or highlighting subsets of the data set is called "brushing" [7, 8].

VizCraft makes it particularly easy to extract regions of interest from the design space. For example, to select a region for which TOGW lies within a certain range, the user can select the circle below the TOGW axis, and then enter the range in the "zoom from" and "zoom to" text fields. This eliminates all design points for which the value of TOGW does not lie within this range. The axis for TOGW is re-calibrated to this new scale, while all other axes retain their calibration. Alternatively, the user can click on any axis, drag the mouse pointer up or down, and release it to zoom into a region of interest. Figure $\mathrm{C} 6$ shows the result of zooming into a region of low TOGW. The text fields at the bottom indicate that there are only 4 design points lying in the region of interest, and that the remaining 1,496 points have been discarded. Since we are interested in designs that yield low values for TOGW, we can now observe other design variables in this design subspace. Perhaps this will allow the designer to gain insight regarding what values of these variables, or what combinations of values of these variables, produced low values of TOGW.

Figure C7 shows the set of constraints corresponding to Figure C6. VizCraft provides application-specific visualization options related to constraint violations. The "no color" option indicates that the polygonal lines representing all the design points are rendered in the default color. The "all" option indicates that the polygonal lines are colored based on the rule that if any constraint is violated for a particular design point, that design point must be rendered in red. If all constraints are satisfied for a particular design point, that design point is rendered in green. In Figure C7 there is no design point that satisfies all constraints. A third option, the "selective" coloring option assigns a color to each polygonal line on the rule that all points for which the selected constraint is violated are colored red, those points for which that constraint is active are colored yellow, and those points for which that constraint is satisfied are colored green.

Finally, VizCraft gives the user an opportunity to highlight any one of the design points. To highlight a design point, the user must click at a point where a polygonal line intersects a vertical axis. Highlighting is done by assigning a bright color to the design point of interest. The highlighted point can also be viewed in its iconic representation in the main window (as in Figure $\mathrm{C1}$ ) by clicking on the "View" button.

\section{CONCLUDING REMARKS}

This paper presented VizCraft, an application for visualizing HSCT designs using parallel coordinates. VizCraft has helped streamline the practice of exploring the effect of design variable combinations on aircraft performance for regions of the design space that have not previously been investigated. Where the designer originally had to manually change design variables in a file, run the analysis code, and then observe the results in a separate plotting package, VizCraft is able to perform these operations with a few button clicks. The data mining capabilities of VizCraft have proved beneficial when large databases of HSCT performance data are available. By using colored driving variables and brushing techniques, designers were able to visually correlate different design variable combinations and/or patterns that resulted in either very good or very bad aircraft performance.

\section{REFERENCES}

1. M. Ankerst, S. Berchtold, and D. Keim, Similarity Clustering of Dimensions for an Enhanced Visualization of Multidimensional Data, Proceedings of IEEE Symposium on Information Visualization (InfoVis'98), Research Triangle Park, NC, October 1998, 52-60.

2. S. Burgee, A.A. Giunta, V. Balabanov, B. Grossman, W.H. Mason, R. Narducci, R.T. Haftka, and L.T. Watson, A Coarse-Grained Parallel Variable-Complexity Multidisciplinary Optimization Paradigm, International Journal of Supercomputer Applications and High Performance Computing, October 1996, 269-299.

3. A. Inselberg, The Plane with Parallel Coordinates, The Visual Computer 1, 1985, 69-91.

4. A. Inselberg, Multidimensional Detective, Proceedings of IEEE Symposium on Information Visualization (InfoVis'97), Phoenix, AZ, October 1997, 100-107.

5. D.L. Knill, A.A. Giunta, C.A. Baker, B. Grossman, W.H. Mason, R.T. Haftka, and L.T. Watson, Response Surface Models Combining Linear and Euler Aerodynamics for Supersonic Transport Design, Journal of Aircraft 36, 1, January-February 1999, 75-86.

6. C.A. Shaffer, D.L. Knill, and L.T. Watson, Visualization for Multiparameter Aircraft Designs, Proceedings of IEEE Visualization'98, Research Triangle Park, NC, October 1998, 491-494.

7. M. O. Ward, A.R. Martin, High Dimensional Brushing for Interactive Exploration of Multivariate Data, Proceedings of IEEE Visualization'95, Los Alamitos, CA, October 1995, 271-278.

8. P.C. Wong and R.D. Bergeron, Multiresolution Multidimensional Wavelet Brushing, Proceedings of IEEE Visualization'96, New York, NY, October 1996, 141148. 


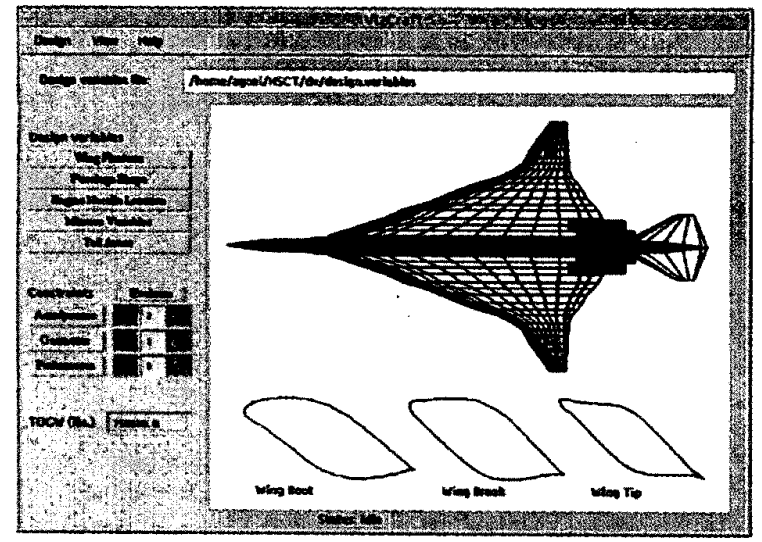

Figure $\mathrm{C} 1$. VizCraft design view window. To make observation easier, the vertical dimension of the wing cross-sections has been magnified.

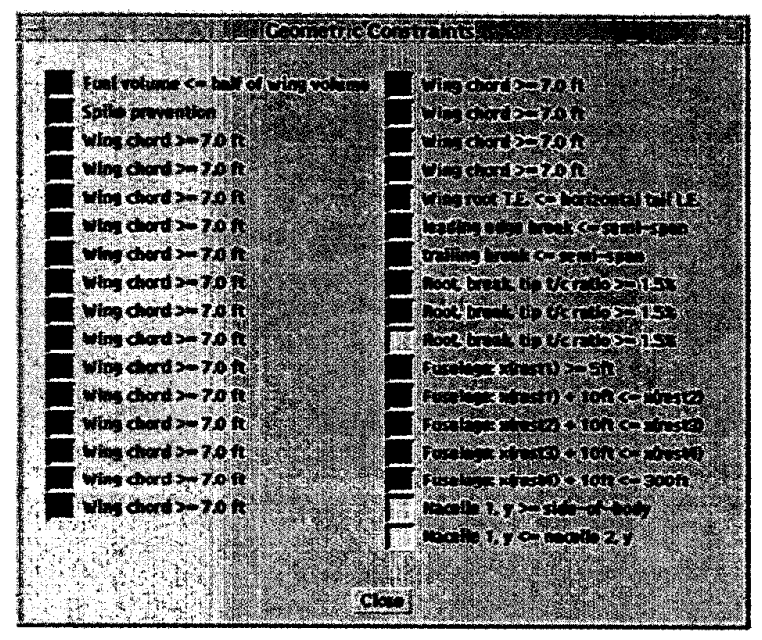

Figure C2. Geometric constraints for one design point.

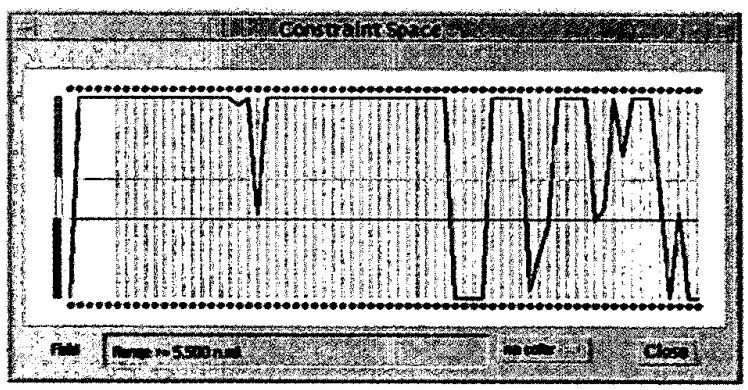

Figure C3. Parallel coordinates representation of constraints for one design point.

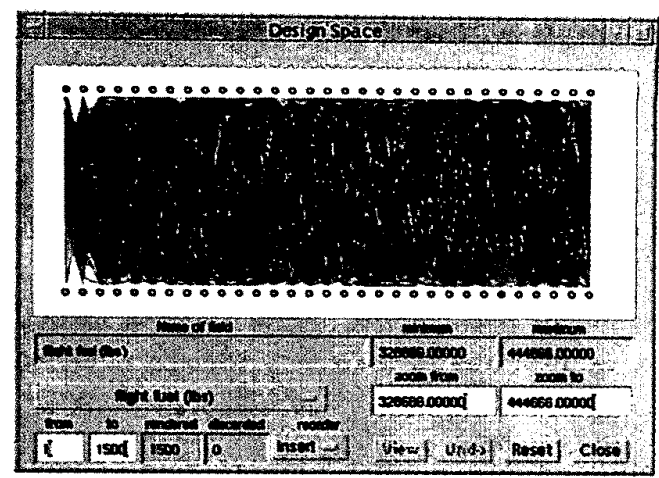

Figure C4. Parallel coordinates representation of 1500 design points.

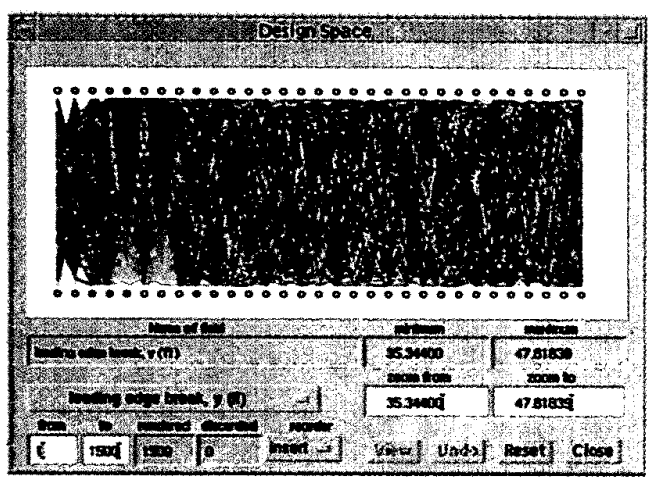

Figure C5. Clever selection of the "color driver" highlights a relationship between two visualization variables.

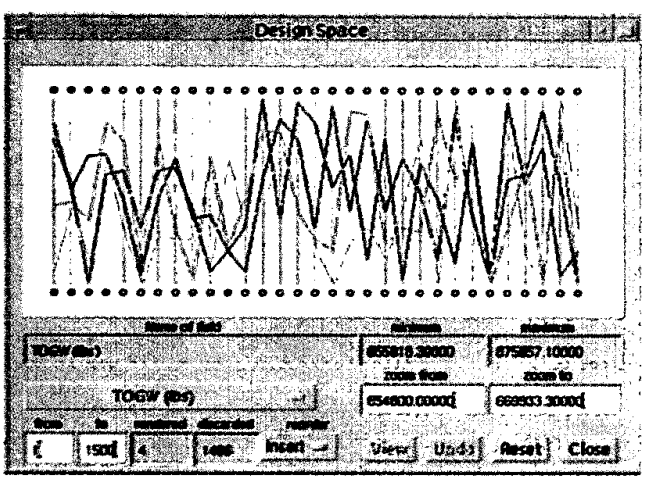

Figure C6. Result of brushing out design points lying outside a certain range of TOGW.

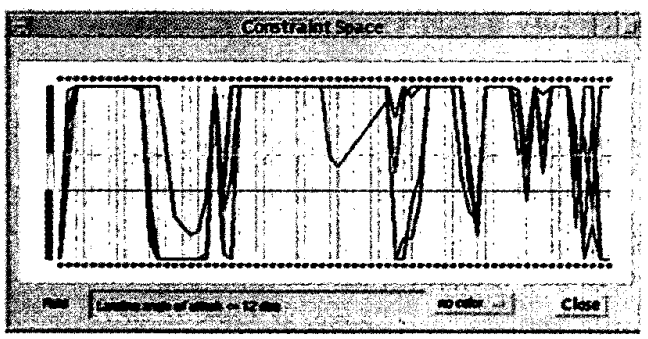

Figure C7. Constraints corresponding to Figure C6.

VizCraft: A Multidimensional Visualization Tool for Aircraft Configuration Design A. Goel, C. Baker, C. A. Shaffer, B. Grossman, R. T. Haftka, W. H. Mason, L. T. Watson 